\title{
Comparative Study on the Uniform Energy Deposition Achievable via Optimized Plasmonic Nanoresonator Distributions
}

\author{
Mária Csete ${ }^{1}$ (1) András Szenes ${ }^{1} \cdot$ Emese Tóth $^{1} \cdot$ Dávid Vass $^{1} \cdot$ Olivér Fekete $^{1} \cdot$ Balázs Bánhelyi $^{2} \cdot$ István Papp $^{3,4}$. \\ Tamás Bíró ${ }^{3}$. László P. Csernai ${ }^{3,4,5} \cdot$ Norbert Kroó 3,6
}

Received: 1 July 2021 / Accepted: 2 December 2021 / Published online: 6 January 2022

(C) The Author(s) 2022

\begin{abstract}
Plasmonic nanoresonators of core-shell composition and nanorod shape were optimized to tune their absorption cross-section maximum to the central wavelength of a short laser pulse. The number density distribution of randomly located nanoresonators along a laser pulse-length scaled target was numerically optimized to maximize the absorptance with the criterion of minimal absorption difference between neighboring layers illuminated by two counter-propagating laser pulses. Wide Gaussian number density distribution of core-shell nanoparticles and nanorods enabled to improve the absorptance with low standard deviation; however, the energy deposited until the overlap of the two laser pulses exhibited a considerable standard deviation. Successive adjustment resulted in narrower Gaussian number density distributions that made it possible to ensure almost uniform distribution of the deposited energy integrated until the maximal overlap of the two laser pulses. While for core-shell nanoparticles the standard deviation of absorptance could be preserved, for the nanorods it was compromised. Considering the larger and polarization independent absorption cross-section as well as the simultaneously achievable smaller standard deviation of absorptance and deposited energy distribution, the core-shell nanoparticles outperform the nanorods both in optimized and adjusted nanoresonator distributions. Exception is the standard deviation of deposited energy distribution considered for the complete layers that is smaller in the adjusted nanorod distribution. Optimization of both nanoresonator distributions has potential applications, where efficient and uniform energy deposition is crucial, including biomedical applications, phase transitions, and even fusion.
\end{abstract}

Keywords Plasmonic nanoresonator · Random distribution · Absorptance improvement · Uniform energy deposition · Numerical optimization

\section{Introduction}

Tailoring the geometry and composition of metal nanoparticles makes it possible to excite localized surface plasmon

Mária Csete

mcsete@physx.u-szeged.hu

1 Department of Optics and Quantum Electronics, University of Szeged, Szeged 6720, Hungary

2 Department of Computational Optimization, University of Szeged, Szeged 6720, Hungary

3 Wigner Research Centre for Physics, Budapest 1121, Hungary

4 Department of Physics and Technology, University of Bergen, 5007 Bergen, Norway

5 Frankfurt Institute for Advanced Studies, 60438 Frankfurt/Main, Germany

6 Hungarian Academy of Sciences, Budapest 1051, Hungary resonances (LSPRs) at specific frequencies. Two widely studied plasmonic nanoparticle types are the dielectric-metal core-shell nanoparticle and the elongated metal nanorod. Each of these plasmonic nanoparticles supports different plasmonic modes that may have advantages and disadvantages depending on application purposes. LSPRs excited on core-shell nanoparticles are hybridized modes of dipolar or higher-order primitive sphere and cavity plasmons [1]. The strength of their interactions depends on the order of both individual modes, the type of hybridization (bonding or anti-bonding) and their interaction-length, that equals to the thickness of the metallic shell. Accordingly, the resonance wavelength of the hybridized modes can be tuned through a 
wide spectral range by the generalized aspect ratio (inner to outer radius ratio, $G A R=r_{1} / r_{2}$ ) of the core-shell nanoparticle $[2,3]$. Another unique property of core-shell nanoparticles is the existence of two distinct GARs, both correspond to nanoresonator compositions that exhibit a dipolar resonance at the same frequency in a specific medium $[4,5]$. The extinction cross-section in thin shell composition originates exclusively from the absorption cross-section, whereas in thick shell composition it is determined by the significantly larger scattering cross-section, in accordance with its larger volume.

On metal nanorods, the LSPRs can be tuned through a wide spectral range by varying their aspect ratio (AR, that is, the ratio of the long to the short axis) $[6,7]$. The advantage of nanorods over core-shell nanoparticles is that the achieved near-field enhancement may be high due to the large curvature of the metallic apexes. However, their resonant response depends on the relative orientation with respect to the exciting electric field polarization, which is a disadvantage. The longitudinal mode can be excited most efficiently at longer wavelength, when the polarization is parallel to the long axis, whereas the transversal mode appears at shorter wavelength in presence of a perpendicular E-field component and is accompanied by smaller absorption cross-section. In both cases the frequency of the plasmon resonance, the characteristics of the accompanying near-field and far-field phenomena depend on the nanorod shape and size, as well as on the material of the nanoresonator and the adjacent medium.

Core-shell particles and nanorods can be used in a very wide range of applications due to the properties of the localized plasmons excitable on their surface. The high sensitivity of the resonant frequency to the ambient medium makes the plasmonic particles good candidates for sensing applications $[8,9]$. In nanoparticle arrays, the enhancement of near-field intensity and the improvement of far-field emission directivity can be exploited to boost and to out-couple the fluorescence of emitters, respectively $[10,11]$. The relatively small radiation efficiency accompanied by significant absorption in the metallic segment is advantageous in biomedical imaging, cancer therapy, catalysis, and photovoltaics [12-14]. The plasmonic modes transfer the light energy directly to electrons, which effect was used in renewable energy technologies' development [15].

The balance of near- and far-field loss channels is a complex function of the nanoresonator configuration; hence, an effective solution to the, e.g., maximal energy deposition, problem makes a numerical optimization indispensable.

In present study, the near-field confinement and losses accompanying plasmons excitation are of particular importance. The absorptance of various targets can be enhanced by embedded plasmonic nanoresonators that can serve as local absorbers throughout their damage threshold. This threshold sensitively depends on the geometry of the nanoresonators and on laser illumination parameters. For nanospheres with a diameter of $30 \mathrm{~nm}$, a damage threshold of $5 \times 10^{12} \mathrm{~W} / \mathrm{cm}^{2}$ was reported for $800 \mathrm{~nm}$ wavelength and $30 \mathrm{fs}$ laser pulses [16]. In comparison, the highest intensity that might result in LSPR excitation without the damage of nanotriangles is $1.4 \times 10^{12} \mathrm{~W} / \mathrm{cm}^{2}$ for $800 \mathrm{~nm}$ wavelength and $42 \mathrm{fs}$ pulses [17]. In case of nanospirals, a larger $8 \times 10^{14} \mathrm{~W} / \mathrm{cm}^{2}$ threshold was described for $1500 \mathrm{~nm}$ and $20 \mathrm{fs}$ laser pulses [18].

Near the damage threshold nonlinear phenomena can arise due to the high field strengths that need to be considered. The second order nonlinearity of gold is usually neglected for symmetry reasons, whereas the third order nonlinear susceptibility $\left(\chi^{(3)}\right)$ is strongly dependent on the geometry of the nano-object, on the wavelength and pulselength of the illumination [19]. Full non-perturbative timedomain hydrodynamic approach of the electron plasma under femtosecond excitation made it possible to describe the nonlinear response of nanostructures. The importance of resonant effects in the enhancement of nonlinear phenomena as well as the role of nanostructures' topology was proven [20]. Nanoparticles with high spatial symmetry allow nonlinear conversion only between localized plasmon modes with the same symmetry properties. Charge separation accompanying dipoles (quadrupoles) at the second harmonic is smaller (larger) than that arising in presence of the fundamental dipolar mode; however, the quadrupole is a dark mode, which makes it concealed in the far-field [21]. Coupling nonlinear hydrodynamic equations with the Maxwell equations enabled to describe the boundary second harmonic (SH) and third harmonic (TH) generation in metallic nanostructures without the need of an a priori assumption about the nonlinear susceptibility values. The $\chi^{(2)}$ and $\chi^{(3)}$ were estimated from the quadratic and cubic dependence of SH and TH intensity for specific nano-objects [22]. The metal was modeled as a two-component medium using the hydrodynamic model to describe free electrons and Lorentz oscillators to account for core electron contributions to both the linear dielectric response and harmonic generation. It was shown that in case of silver nanopillars illuminated with $800 \mathrm{~nm}$ and $20 \mathrm{fs}$ pulse the transverse magnetic SH (TH) generation is enhanced (decreased) by bound charges [23].

The implantation of solid plasmonic nanoparticles and plasmonic nanoshells into fusion ignition targets has been already proposed $[24,25]$. The primary studies revealed that the large electromagnetic field enhancement around such nanoresonators is capable of triggering the nuclear chain reactions in conventional deuterated polystyrene fuel targets [24]. It was demonstrated that the coating of the conventional Pd-based fuel materials with nanoparticles and nanoshells has the potential to overcome the Coulomb barriers and to promote condensed matter fusion reaction [25]. Later, application of ordered nanowire arrays was proposed 
to transfer material into the ultra-hot plasma regime with fs laser pulses of moderate energy [26]. Formation of Z-pinches was demonstrated by irradiating nanowires with fs laser pulses of relativistic intensity [27]. The possibility to promote the energy penetration via nanowires and to enhance the plasma extension was also demonstrated [28].

In our previous studies, we have proposed a novel approach to promote uniform energy deposition and to ensure time-like ignition of fusion targets by doping them with metal nanoparticles [29]. Energy deposition increased by plasmonic nanoparticles allows the initial compression of the fusion target to be reduced, thus avoiding RayleighTaylor instability that prevents the achievement of inertial confinement fusion [30].

It has also been shown that time-like ignition can be achieved via double-sided irradiation with short and intense laser pulses even at reduced compression, when the absorption of a target is improved by orders of magnitude due to embedded plasmonic core-shell nanoparticles [29]. Beside the energy deposition the charge separation on nanoparticles is also an important aspect, since its amplitude might be commensurate with that accompanying the laser wake field (LWF) dense plasma waves initiated by two intense counter-propagating laser pulses [31]. In this study, we have exploited the large absorption cross-section provided by the dipolar resonance on the core-shell nanoparticles of thin shell composition and by the dipolar resonance on nanorods. We have optimized the geometry of individual nanoresonators as well as their distribution to achieve efficient and uniform energy deposition in fusion model-targets.

Various numerical solution techniques have been developed for physical [32] and chemical problems [33, 34] that are based on different optimization techniques. Stochastic, deterministic, and hybrid optimization methods have been also implemented to solve such complex problems. In recent years, nanophotonical systems have been optimized by using our in-house developed GLOBAL algorithm [35]. It has already proven its robustness in frequency and timedomain problems as well; among the optimized phenomena are single-photon detection efficiency maximization and few-cycle plasmonic pulse-length minimization [36, 37]. Evaluation of the complex objective function above a multi-dimensional parameter-space in case of a mesoscopic target consisted of nanoscaled resonators requires a lot of resources; accordingly, a prediction method has been used in present optimization. The applied multilayer absorption law covering method is similar to the SVR or adaptive neurofuzzy predictor methods in the literature, which are used to optimize the efficiency of wind turbines, the quality of laser welding or to predict and control the strength of robotic finger contact forces [38-40].

The specific purposes were as follows: (i) to improve the absorptance of a polymer slab by using embedded plasmonic nanoresonators of a core-shell composition and a nanorod shape, (ii) to determine such number density distributions that are capable of ensuring uniform distribution of deposited energy integrated until the overlap of two counter-propagating short laser pulses that are used for the illumination of a model-target with a pulse-length scaled size, and (iii) to map the charge separation that accompanies the plasmonic resonance near the damage threshold of these nanoresonators.

\section{Methods}

The optical cross-sections (OCS: absorption cross-section $(A C S)$, scattering cross-section (SCS), extinction crosssection $(E C S)$ ) of the silica-gold core-shell nanoparticle $\left(r_{1}=25.5 \mathrm{~nm}\right.$ inner and $r_{2}=30 \mathrm{~nm}$ outer radii) and gold nanorod ( $a_{\text {long }}=75 \mathrm{~nm}$ long and $a_{\text {short }}=25 \mathrm{~nm}$ short axes) acting as a plasmonic nanoresonator at the central wavelength $(795 \mathrm{~nm})$ of the target illumination were determined by total-field-scattered-field (TFSF) procedure in finite element method (FEM), namely, via RF module of COMSOL Multiphysics (Fig. 1a, c, d). In case of the core-shell nanoparticle, the thin shell composition was selected, since this results in an extinction dominated by large absorption [4].
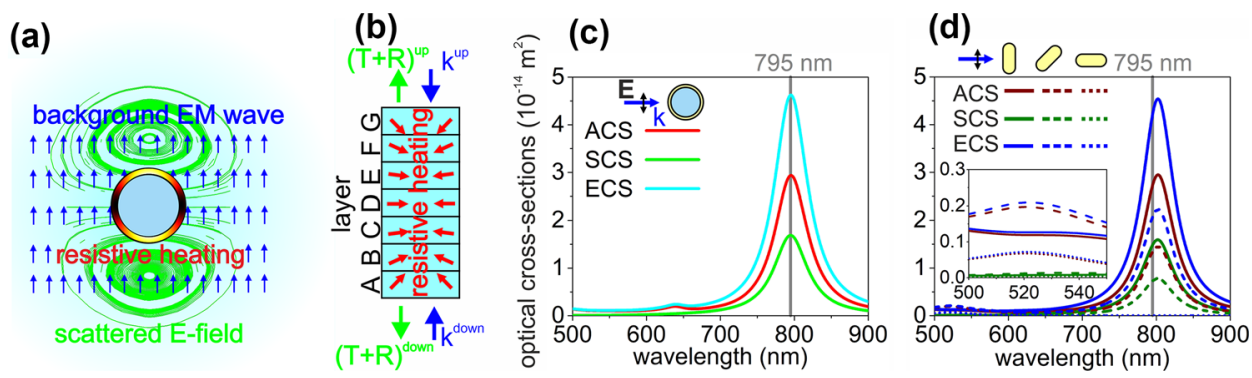

Fig. 1 a TFSF formulation used to determine the $O C S$ of nanoresonators. b Illumination of the targets by two counter-propagating pulses to extract the full optical response and to determine the absorptance.
Orientation (in)dependent $O C S$ spectra of a resonant individual c core-shell nanoparticle and $\mathbf{d}$ nanorod in polymer medium 
These nanoresonators were embedded into a polymer target; the index of refraction of this medium used in simulations was determined by ellipsometry. The wavelength dependence of the real part of the refractive index was considered (at the central wavelength it is 1.53), whereas the imaginary part was neglected, according to the negligible polymer absorptance.

The analogous nanoresonator number densities $(\rho)$ of core-shell nanoparticles and nanorods were determined based on the ratio of their ACS, by considering that the absorption coefficient satisfies the $\alpha=A C S * \rho$ relation, when low number density of nanoresonators is supposed.

To determine the absorptance and absorption coefficient, a supercell with typically $1 \mu \mathrm{m}$ side-length was used, and the $21 \mu \mathrm{m}$ long target was divided into 7 segments, each of $3 \mu \mathrm{m}$ thick along the laser pulses propagation direction, and inside them the number density of randomly distributed nanoresonators was different (Fig. 1b). The lateral size of the supercells was selected to ensure that the nanoresonator number density is variable in neighboring layers with a reasonable resolution, but was not matched intentionally to the wavelength in the medium, in order to avoid artificial coupling effects. These supercells were illuminated by two counter-propagating linearly polarized short laser pulses injected through internal ports. At the vertical sides, periodic boundary conditions were used, whereas behind the horizontal ports PML layers were inserted to eliminate spurious reflections. The subwavelength metal nanoresonators require fine meshing in the order of half skin-depth, whereas the three-dimensional model-targets enable coarser meshing in the order of a fifth-wavelength along the several micrometer long target. These simultaneous mesh scale-regions result in a computation demand in the order of 128-256 GB of RAM.

The transmittance and reflectance were extracted at interfaces inserted between the ports and PML layers, and during evaluation of the absorption coefficient the equivalence of the two opposite pulse propagation directions was supposed. The $21 \mu \mathrm{m}$ target length corresponds to the full-width-athalf-maximum (FWHM) of a 120 fs laser pulse envelope. A target with this length is illuminated most efficiently by the two counter-propagating pulses, when they overlap at $240 \mathrm{fs}$ at the middle of the target.

The primary purpose was to improve the absorptance; accordingly, the nanoresonator number density was varied in the $\rho \sim 1-15 \mu \mathrm{m}^{-3}$ interval. Before optimization, various distributions of the nanoresonators were tested, among them uniform (Fig. 2), single, and double-peaked Gaussian distributions (Fig. 3). Then, first, the nanoresonator distribution was optimized in steady-state RF module with the objective function of maximal absorption and with the criterion of minimal absorption difference between neighboring layers (Figs. 4 and 5). During optimization, tendencies of the achieved absorptance and its standard deviation $(\delta)$ were monitored and the number density distribution was varied by moving nanoresonators in between layers to increase absorptance and to decrease $\delta$. The in-house developed algorithm was combined with a prediction method, which relies on statistical sampling of preceding random distributions and successive approximation of the optimal solution by considering their standard deviation that enables to improve gradually the random parameters in succeeding solutions [34-37]. The stochastic effect of the nanoresonator's location distribution at a specific number density distribution was treated by fitting the Lambert-Beer law with multiple parameters. This procedure is still time-consuming, due to the slow model evaluation with the specific objective function completion takes typically days by using 256 GB of RAM.

Second, the steady-state absorptance and the average standard deviation of absorptance sampled in different layers were determined by inspecting several random location distributions of the previously optimized nanoresonator number density distribution. Third, a representative system exhibiting a standard deviation of absorptance that approximates the average $\delta$ of the specific nanoresonator number density distribution was selected (Figs. 4 and 5/a, c). To diminish the difference that can arise between symmetrically aligned layers embedding the same number of nanoresonators in case of arbitrary random location distributions, symmetrically arranged nanoresonator locations were supposed in analogous layer pairs (A-G, B-F, C-E) (Fig. 1b). We can suppose that the location distribution possesses a mirror or even a central symmetry without the loss of generality (Figs. 4 and 5 inset).

Fourth, the energy and energy density, as well as the power loss and power loss density distribution, were determined and analyzed separately for the multitudes of nanoresonators in each layer as a function of time (Figs. 4 and 5/e, g/a, b). Furthermore, the time dependence of the energy and energy density were determined not only in the nanoresonators but also in the complete layers, since the confined near-field has the potential to interact with the surrounding media in general case (Figs. 4 and 5/e). To ensure uniform, e.g., phase transition or ignition, the deposited energy integrated until the time instant, when the laser pulses overlap, must be balanced in different layers. Accordingly, as a fifth step the integrated energy and energy density as well as the power loss and power loss density distribution were integrated throughout the maximal overlap of the counter-propagating pulses (Figs. 4 and 5/e, $\mathrm{g} / \mathrm{c}-\mathrm{f})$. Finally, the nanoresonator number density distribution was adjusted to ensure a standard deviation in deposited energy integrated until 240 fs commensurate with (or even smaller than) the typical $\delta$ of absorption (Figs. 4 and 5/b, d; f, h/a-f).

To determine the time-evolution of the charge separation accompanying the short laser pulse illumination of the resonant core-shell nanoparticles and nanorods, the hydrodynamic equations coupled with the Maxwell 
equations were implemented into the transient RF module of COMSOL Multiphysics (Fig. 6). The interaction of plasmonic nanoresonators with a 120 fs laser pulse was described via the wave equation including the induced polarization:

$\nabla \times \nabla \times \boldsymbol{E}(\boldsymbol{r}, t)+\frac{1}{c^{2}} \partial_{t t} \boldsymbol{E}(\boldsymbol{r}, t)+\mu_{0} \partial_{t t} \boldsymbol{P}(\boldsymbol{r}, t)=0$

Here $\boldsymbol{E}(\boldsymbol{r}, t)$ is the electric field with full spatio-temporal dependence, $\mu_{0}$ is the vacuum permeability, and $\boldsymbol{P}(\boldsymbol{r}, t)$ is the induced polarization. The time-dependence of the induced polarization was defined with the macroscopic spatially dependent electron density $\left(n_{e}\right)$ and electron velocity $\left(\boldsymbol{v}_{e}\right)$ accounting for the polarization currents:

$\partial_{t} \boldsymbol{P}(\boldsymbol{r}, t)=-e n_{e} \boldsymbol{v}_{e}$

which couples the Maxwell equations to the hydrodynamic equations as follows:

$m_{e} n_{e}\left(\partial_{t} \boldsymbol{v}_{e}+\boldsymbol{v}_{e} \cdot \nabla \boldsymbol{v}_{e}\right)+\gamma m_{e} n_{e} \boldsymbol{v}_{e}=-e n_{e}\left(\boldsymbol{E}+v_{e} \times \boldsymbol{H}\right)$

$\partial_{t} n_{e}+\nabla \cdot\left(n_{e} v_{e}\right)=0$

where $\gamma$ is a phenomenological coefficient related to damping, while $e$ and $m_{e}$ are the charge and mass of electron, respectively.

\section{Results}

\section{Optical Cross-section of Core-Shell Nanoparticles and Nanorods Resonant at the Central Wavelength of the Illuminating Laser Pulse}

Via TFSF formulation, the complete optical cross-section of the plasmonic nanoresonators was determined, including the absorption, scattering, and extinction cross-sections (Fig, $1 \mathrm{a}, \mathrm{c}, \mathrm{d})$. Considering that the purpose is a large and uniform energy deposition throughout the target, the absorption cross-section of individual nanoresonators is the most relevant quantity; accordingly, this was compared quantitatively for the core-shell nanoparticle and nanorod type nanoresonators.

At $795 \mathrm{~nm}$ central wavelength of the short laser pulse, the resonant core-shell nanoparticle exhibits $2.97 \times 10^{-10}$ $\mathrm{cm}^{2}$ absorption cross-section, whereas the ACS of the resonant nanorod is $2.75 / 1.36 / 0.002 / 0.758 \times 10^{-10} \mathrm{~cm}^{2}$ in $0 \% 145^{\circ} / 90 \%$ averaged random orientation of the long axis with respect to the $\mathbf{E}$-field oscillation direction of the illuminating laser pulse. These values correspond to absorption cross-section ratio of $A C S_{\text {core-shell }} / A C S_{\text {nanorod }}=1.08 / 2$. $18 / 1490 / 3.91$, which indicates that the core-shell nanoparticle exhibits a slightly larger ACS than the nanorod, even if it is perfectly aligned to support longitudinal plasmon resonance. Accordingly, it is expected that a core-shell nanoparticle distribution is capable of resulting in $~ 4$-times larger absorptance than randomly oriented nanorods, when the same number density is applied inside the targets.

\section{Comparison of Uniform Nanoresonator Distributions}

The illumination of a target containing uniform number density but random location distribution of nanoresonators resulted in an Ohmic loss, which decreases exponentially towards the middle of the sample, in accordance with the Lambert-Beer law.

Moreover, the Ohmic loss decreases symmetrically, when the number density distribution of nanoresonators is symmetrical inside a target illuminated synchronously by two counter-propagating laser pulses (Fig. 2).
Fig. 2 Representative uniform number density distributions of a 70 core-shell nanoparticles and $\mathbf{d} 70$ oriented nanorods, and $\mathbf{b}, \mathbf{e}$ and $\mathbf{c}, \mathbf{f} 70$ and 280 randomly oriented nanorods in a $1 \times 1 \times 21 \mu^{3}$ supercell of polymer target, all with random location distributions
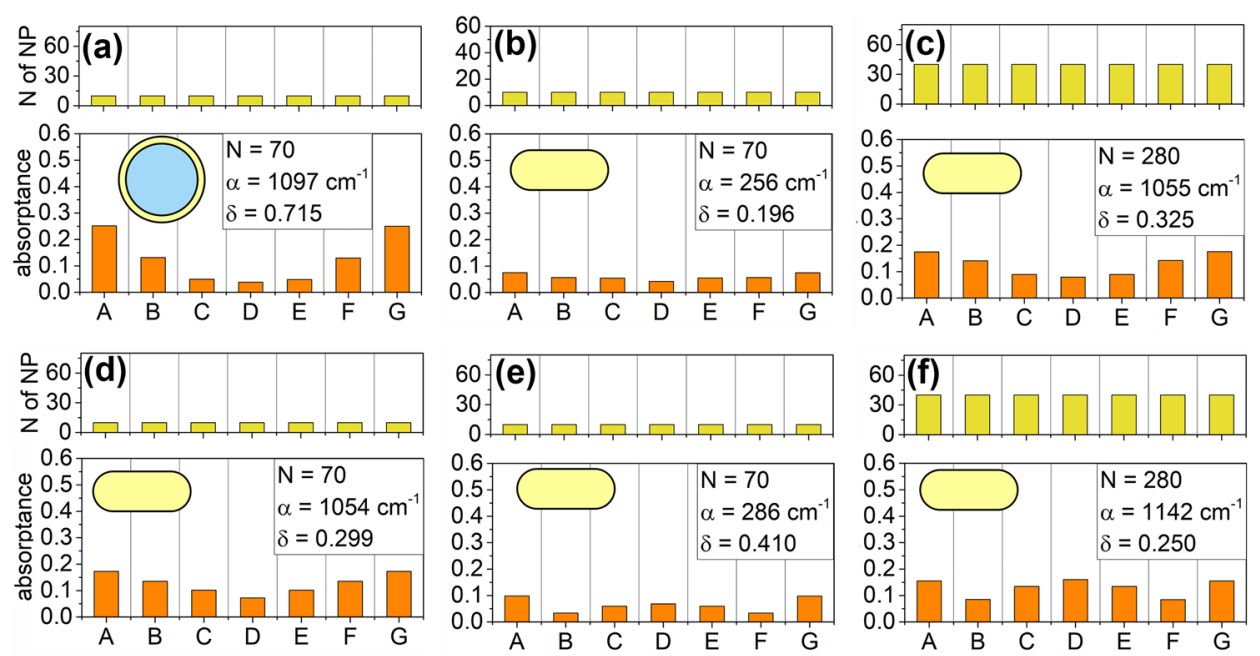
The uniform number density distribution of randomly located core-shell nanoparticles and oriented nanorods results in one single dip in the Ohmic loss distribution at the center of the target (Fig. 2a, d). In contrast, the uniform number density distribution of randomly located and oriented nanorods promotes the appearance of either one or two minima in the Ohmic loss distribution (Fig. 2b, c and $e, f)$. The appearance of one and double dips depends on the specific location and orientation distribution. When two minima appear, they are symmetrically arranged with respect to the target center, due to the predefined symmetrical nanoresonator location distribution. The probability of two extrema is larger in case of nanorods due to the orientation dependence of their ACS. Namely, the achieved absorption correlates with the average of the $\mathbf{E}$-field projection onto the nanorod long axes that correlates with the fraction of the longitudinal modes.

The parallel (randomly) oriented nanorods result in an absorptance, which approximates (is smaller than) the absorptance achievable via core-shell nanoparticles. The ratio of absorptances is in accordance with the ratio of the $A C S_{\text {core-shell }} / A C S_{\text {nanorod, }}$ when the same number density is applied (Fig. 2a-to-b, d, e). By applying the same 70 number of oriented (random) nanorods in a $1 \times 1 \times 21 \mu \mathrm{m}^{3}$ supercell of polymer target, the achieved absorptance is almost the same (four-times smaller), as a result of the almost unity ( four) $A C S_{\text {core-shell }} / A C S_{\text {nanorod }}$ ratio (Fig. 2a-to-d (b, e)). Accordingly, by inserting 4-times larger (280) number of randomly oriented nanorods into the same volume, the achieved absorptance is almost equal to that reached via (70) core-shell nanoparticles (Fig. 2a-to-c, f).

Based on the significantly larger and polarization independent absorptance, the core-shell nanoresonator type is qualified as a better choice; however, the standard deviation of absorptance is typically several-times larger (Fig. 2a to b-f).

The illumination of nanorods aligned along the $\mathbf{E}$-field oscillation direction resulted in an absorptance commensurate with that achievable via core-shell nanoparticles. However, the standard deviation of absorptance is similar to the average $\delta$ achievable via the same number of randomly oriented nanorods (Fig. 2d-to-b, e). Hence, the random orientation of the nanorods enables to preserve the smaller standard deviation of absorptance on the average.

\section{Comparison of Different Gaussian Distributions}

Different Gaussian number density distributions of nanoresonators were compared, which exhibit single or double peaks in the absorptance distributions (Fig. 3). When the same number of nanoresonators was embedded into the target, the ratio of the achieved absorptances was in accordance with the ratio of the $A C S_{\text {core-shell }} / A C S_{\text {nanorod }}$, independently of the distribution type. Accordingly, the 4-times larger (280) number of nanorods resulted in almost the same $\left(1138 \mathrm{~cm}^{-1}\right.$ and $1103 \mathrm{~cm}^{-1} ; 1092 \mathrm{~cm}^{-1}$ and $\left.1140 \mathrm{~cm}^{-1}\right)$ absorption, as the (70) core-shell nanoparticles $\left(1136 \mathrm{~cm}^{-1}\right.$ and $1025 \mathrm{~cm}^{-1}$; $1102 \mathrm{~cm}^{-1}$ and $1090 \mathrm{~cm}^{-1}$ ) in case of wide and narrow single-peaked Gaussian, and small and large amplitude double-peaked Gaussian number density distributions (Fig. 3 a, c and b, d; e, g and f, h).

However, the relation of the standard deviation of absorptance is nanoresonator distribution type dependent: it is smaller (i) for core-shell nanoparticles and nanorods, when the single-peaked Gaussian distribution is wider and (ii) for core-shells (nanorods), when the double-peaked Gaussian distribution has smaller (larger) amplitude. For
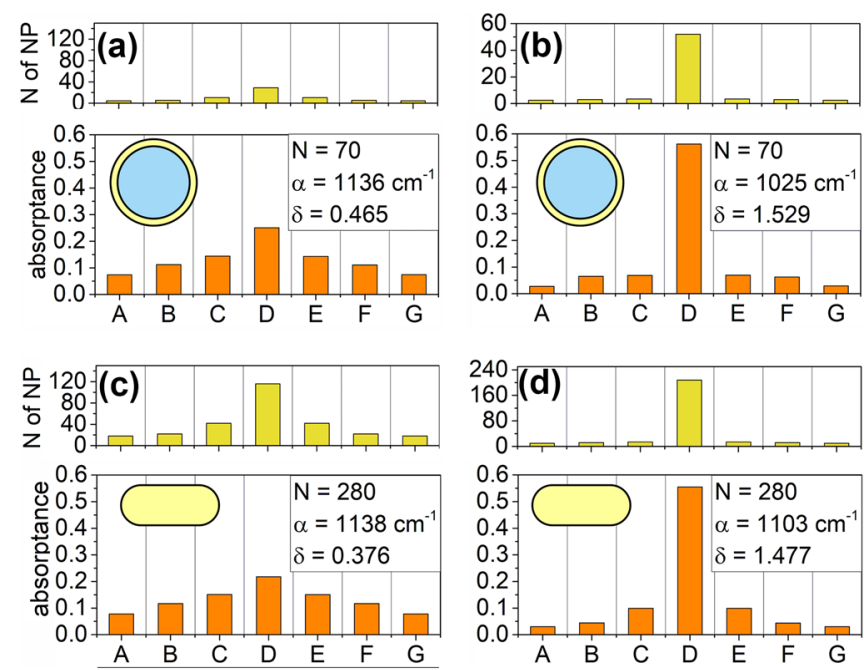

Fig. 3 Comparison of different Gaussian nanoresonator number density distributions: a, c wide and b, d narrow single-peaked Gaussian and $\mathbf{e}, \mathbf{g}$ small and $\mathbf{f}, \mathbf{h}$ large amplitude double-peaked Gaussian dis-
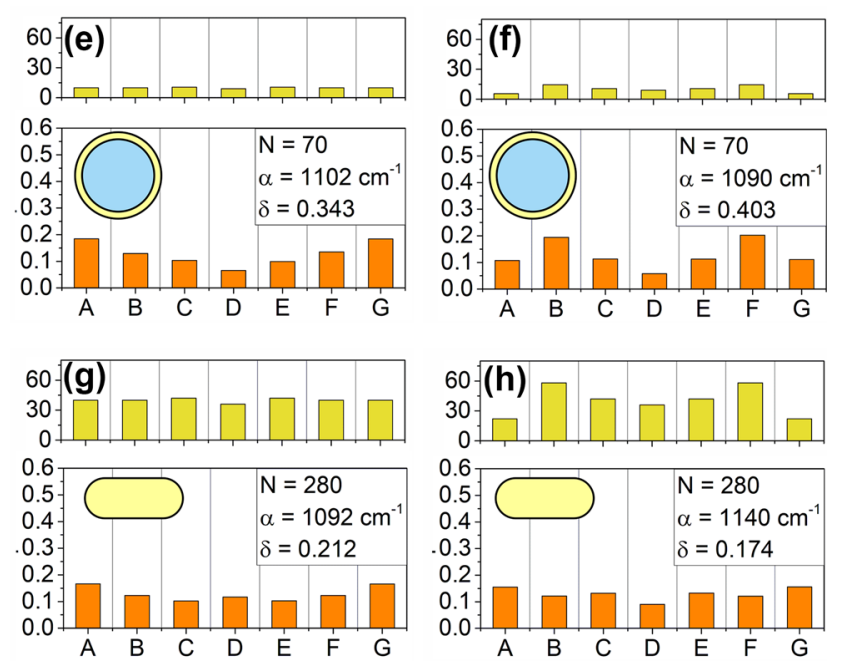

tribution of $\mathbf{a}, \mathbf{b}, \mathbf{e}, \mathbf{f}$ core-shell nanoparticles and $\mathbf{c}, \mathbf{d}, \mathbf{g}, \mathbf{h}$ randomly oriented nanorods, all with random nanoresonator location distributions 
nanorods, the orientation dependence enables to achieve larger absorptance with smaller standard deviation via larger amplitude double-peaked Gaussian nanoresonator distribution.

Analogous 70 and 280 nanoresonator numbers in a $1 \times 1 \times 21 \mu \mathrm{m}^{3}$ supercell of polymer target result in commensurate absorptance, and both the single-peaked and the double-peaked distribution exhibit a slightly larger standard deviation of absorptance for core-shells.

The wide single-peaked Gaussian distribution was selected as the primary distribution for steady-state optimization since it is experimentally more feasible. Moreover, this makes it possible to reach larger absorption, than the narrow single-peaked or either double-peaked Gaussian distributions (except the large amplitude double-peaked nanorod distribution, which shows similar absorption). Its standard deviation is smaller than for narrow single-peaked Gaussian distribution, and it is just slightly larger than the $\delta$ in any inspected double-peaked distributions, nanoresonator type independently.

\section{Comparison of Optimized and Adjusted Gaussian Distributions}

The energy and energy density as well as the power loss and power loss density were inspected in details for the multitudes of nanoresonators in all layers as a function of time (Figs. 4 and 5).

When these quantities are analyzed for both nanoresonators (for the core-shell nanoparticle), one has to consider several phenomena. (i) When the nanoresonator multitudes are considered separately, both the energy and power loss tendencies differ from the corresponding energy density and power loss density tendencies along the layers either for the instantaneous values, or for the values integrated throughout the specific time instant of pulse-peaks overlap. This is due to that normalization is performed by division via the sum of nanoresonator volumes in the specific layers; however, this total volume differs in the neighboring layers in the inspected cases. (ii) The energy in the nanoresonator is smaller than in the full layer (but larger than in the shell), since the light energy is non-zero in the polymer (and in the silica core). (iii) The power loss in the nanoresonator equals with that in the full layer (moreover, it equals with that in the shells), since the polymer model-target medium (and the silica core) is non-absorbing. Accordingly, tendencies in energy and power loss as well as in their densities in nanoresonators are analogous for nanorods (differ for core-shells) along the layers (however, this difference is small due to the small volume fraction of the silica core in core-shell nanoparticles).
We present these quantities for the multitudes of nanoresonators separately for each layer, and for energy and energy density the complete layers are also considered. The standard deviation is the same for the energy and energy density, when integration is realized over the layers, since the volume of the layers is the same.

The primarily optimized core-shell distribution determined by minimizing the absorptance difference between layers consisted of 72 core-shell nanoparticles in a $1 \times 1 \times 21$ $\mu \mathrm{m}^{3}$ supercell of the polymer model-target and exhibited an $\alpha_{\text {avg }}=892 \mathrm{~cm}^{-1}$ average absorption coefficient, $A_{\text {avg }}=0.846$ average absorptance with $\delta_{\text {avg }}=0.102$ average standard deviation of absorptance (Fig. 4a, c: left columns). A representative random location distribution of core-shell resonators having an $\alpha=958 \mathrm{~cm}^{-1}$ absorption coefficient, $A=0.866$ absorptance with $\delta=0.093$ standard deviation of absorptance, that are commensurate with the averaged values, was studied in time-domain (Fig. 4a, c: right column).

All inspected quantities start to increase at the beginning of short-pulse illumination then exhibit a global maximum (Fig. 4ea, eb, ga, gb). There is a well-defined difference between energy (power loss) and energy density (power loss density) in different neighboring layers (Fig. 4ea (ga) and eb (gb)).

The difference between layers increases with time until a layer specific delay. At the average of these delays (240 fs) that corresponds to the complete overlap of the two counter-propagating pulses at the target center, the standard deviation of energy (power loss) and energy density (power loss density) are $\delta_{\text {energy }}=0.387\left(\delta_{\text {power_loss }}=0.408\right.$ ) and $\delta_{\text {energy_density }}=0.541 \quad\left(\delta_{\text {power_loss_density }}=0.567\right)$, respectively, which indicate 4.2 (4.4)- and 5.8 (6.1)fold increase in standard deviation compared to that of the absorptance distribution (Fig. 4ee (ge) and ef (gf)). When the complete layer is considered in the integration, the standard deviation of energy and energy density $\delta_{\text {energy_layer }}=\delta_{\text {energy_density_layer }}=0.151$ is reduced compared to the multitude of nanoparticles, because the nanoparticle distribution becomes less dominant (Fig. 4ee and ef).

The successive modification of the number density distribution along the layers made it possible to improve the core-shell nanoresonator doped target characteristics. The adjusted distribution exhibited an $\alpha_{a v g}=1094 \mathrm{~cm}^{-1}$ average absorption coefficient, $A_{\text {avg }}=0.896$ average absorptance with $\delta_{\text {avg }}=0.412$ average standard deviation of absorptance (Fig. 4b, d: left columns). For a representative system, even larger $\alpha=1152 \mathrm{~cm}^{-1}$ absorption coefficient and larger $A=0.911$ absorptance with standard deviation of $\delta=0.092$ is reached that is slightly smaller, when compared to the original wide Gaussian distribution determined via steadystate computations (Fig. 4b, d right columns).

Time-dependence of all inspected quantities is similar to those in the primarily optimized core-shell distribution, 
Fig. 4 a, b Centrally symmetric a primarily optimized and $\mathbf{b}$ adjusted core-shell Gaussian number density distribution, the corresponding $\mathbf{c}, \mathbf{d}$ average absorptance distribution of several random distributions (left column), and of a representative distribution with a standard deviation approximating the average $\delta$ of absorptance (right column). The corresponding energy/energy density in coreshell nanoparticles as well as in the complete layers, and the power loss/power loss density distribution in core-shell nanoparticles in different layers ea, fa/eb, fb and ga, ha/gb, $\mathbf{h b}$ as a function of time and ec, fc/ed, fd and gc, hc/gd, hd integrated throughout different time-intervals and ee, fe/ef, $\mathbf{f f}$ and ge, he/gf, hf the distribution of values integrated in different layers until $240 \mathrm{fs}$. The dotted and solid rendering refers to, when the values were monitored in the core-shell and full layers, respectively. Inset: schematics of symmetric core-shell distributions in the target
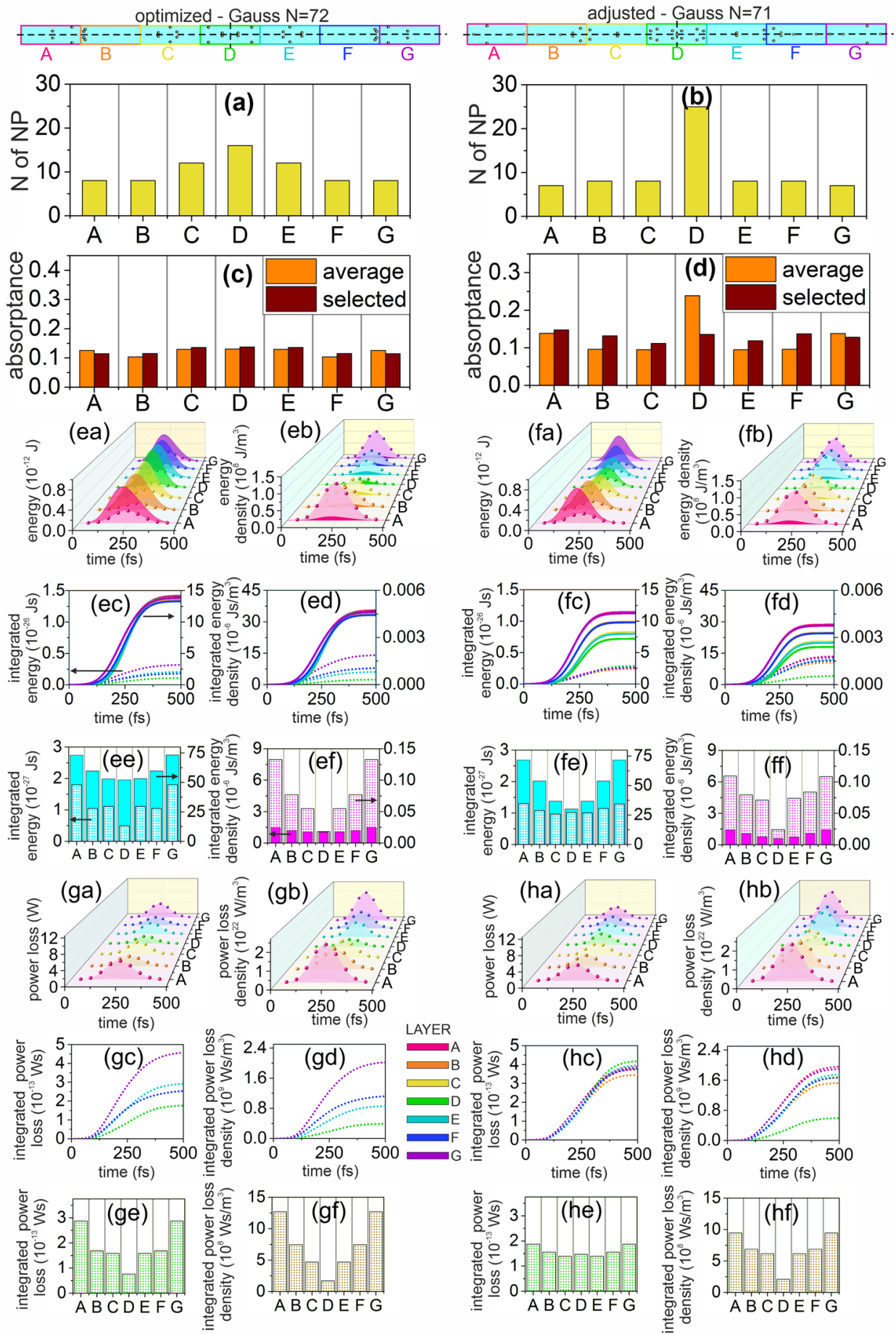

namely, they exhibit global maxima, but these are less distinguishable in succeeding layers after adjustment (Fig. 4fa, $\mathrm{fb}$, ha, hb to ea, eb, ga, gb). The standard deviation of energy (energy density) is 8.1-times (almost 1.8-times) smaller, namely, $\delta_{\text {energy }}=0.048\left(\delta_{\text {energy_density }}=0.3\right)$ is achieved, compared to the values in primarily optimized distribution (Fig. 4fa, fc, fe (fb, fd, ff)).
The standard deviation of power loss (power loss density) is $\delta_{\text {power_loss }}=0.130\left(\delta_{\text {power_loss_density }}=0.371\right)$, which is 3.1-times (1.5-times) smaller than the value in case of the primarily optimized distribution (Fig. 4ha, hc, he (hb, hd, hf)). The standard deviation of the energy and energy density integrated in the complete layer becomes larger than in the multitude of nanoparticles, namely, 
Fig. 5 a, b Centrally symmetric a primarily optimized and b adjusted nanorod Gaussian number density distributions, the corresponding $\mathbf{c}, \mathbf{d}$ average absorptance distributions of several random distributions (left column), and of a representative distribution with a standard deviation approximating the average $\delta$ of absorptance (right column). The corresponding energy/energy density in nanorod as well as in the complete layers, and the power loss/ power loss density distribution in nanorods in different layers ea, fa/eb, fb and ga, ha/gb, $\mathbf{h b}$ as a function of time and ec, fc/ed, fd and gc, hc/gd, hd integrated throughout different time-intervals and ee, fe/ef, $\mathbf{f f}$ and ge, he/gf, hf the distribution of values integrated in different layers until $240 \mathrm{fs}$. The dotted and solid rendering refers to, when the values were monitored in the nanorods and full layers, respectively. Inset: schematics of the symmetric nanorod distributions in the target
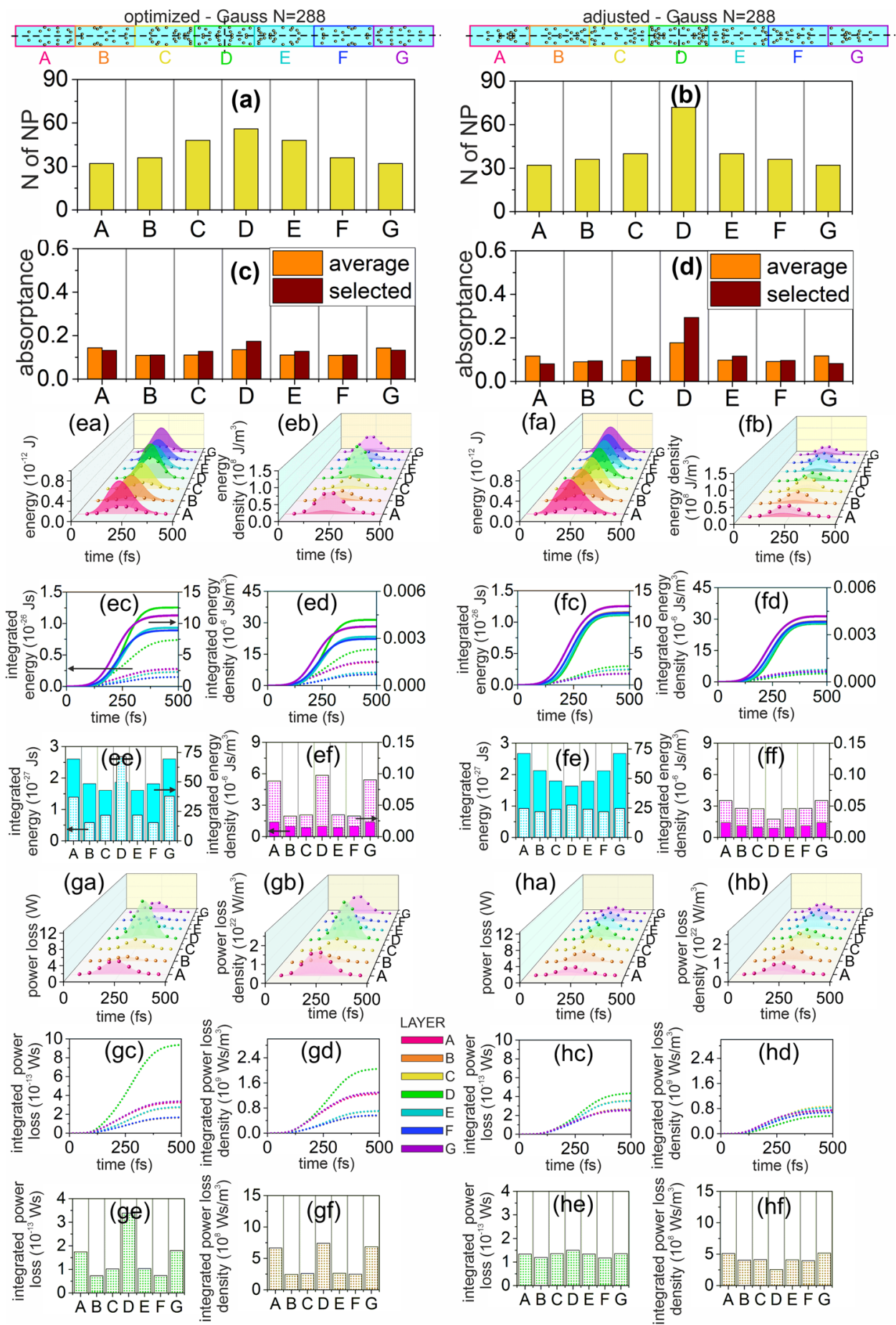

$\delta_{\text {energy_layer }}=\delta_{\text {energy_density_layer }}=0.334$ (Fig. 4fe and ff). This is a result of the simultaneous energy capture inside and around the core-shell nanoparticles in case of the specific location distribution selected from the manifolds of adjusted narrow Gaussian number density distributions.

The integrated energy (power loss) rapidly increase over time in the adjusted core-shell distribution as well (Fig. 4fc, fd (hc, hd)-to-ec, ed (gc, gd), fe, ff (he, hf)-to-ee, ef (ge, gf)). However, due to the successively minimized standard deviation in integrated energy in nanoparticles, the rate of this increase is similar in neighboring layers, which ensure more uniform energy dissipation throughout the complete target (Fig. 4fc, fe). For the energy density the difference in time-dependence and the resulted $\delta$ is significantly larger according to the different core-shell nanoparticle number density in the neighboring layers (Fig. 4fd, ff). The largest 


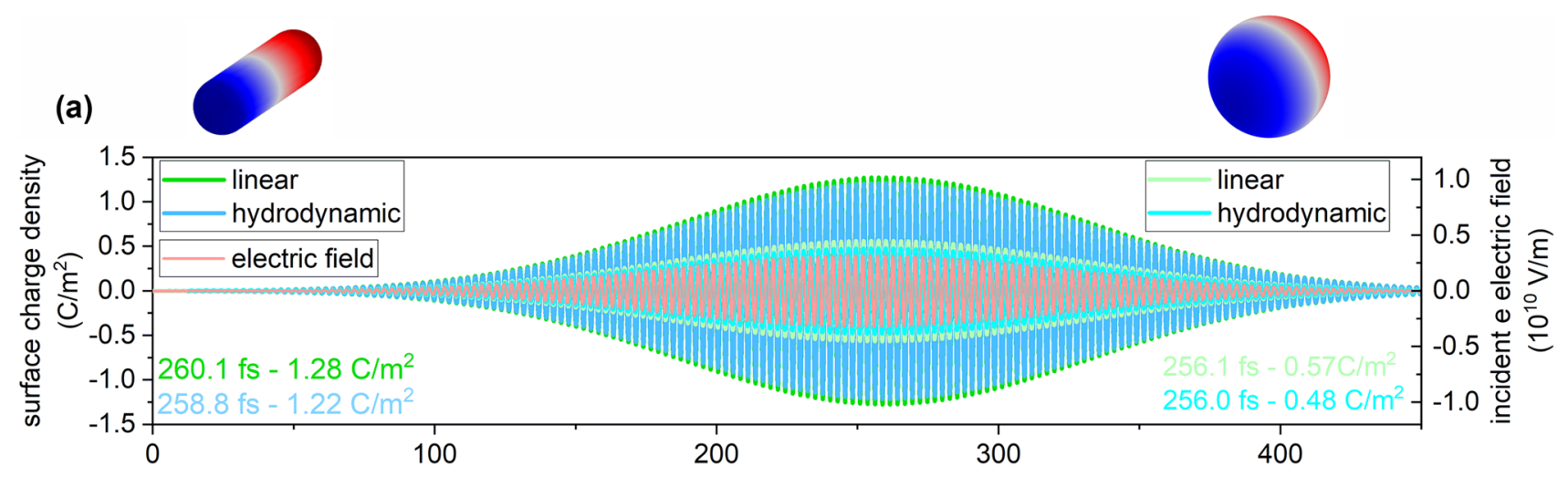

time (fs)

(b)

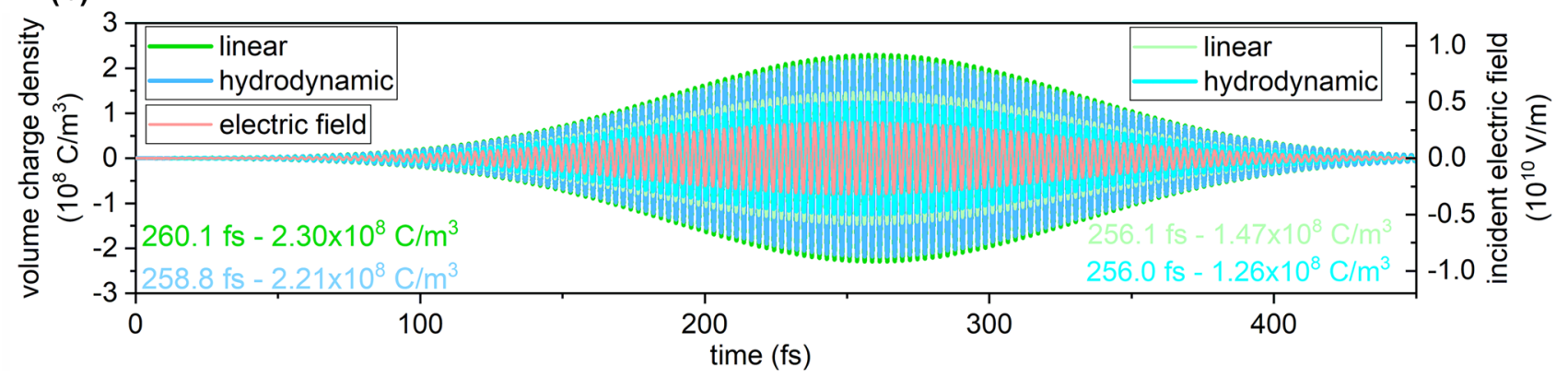

Fig. 6 a Surface and b corresponding volume charge separation accompanying the plasmonic resonance induced on the core-shell nanoparticle and nanorod illuminated by $120 \mathrm{fs}$ short pulse of $795 \mathrm{~nm}$ central wavelength at the damage threshold

deposited energy density contrast appears between the central layers of the target, where the nanoparticle density is the highest (Fig. 4ff). The power loss and power loss density exhibit similar tendencies according to the slight difference related to the energy in the core (Fig. 4h). When the energy is integrated over the complete layer, the energy and energy density decrease exponentially towards the center of the target, with a larger slope in the adjusted case (Fig. 4fe and ff).

The primary nanorod distribution was determined similarly by maximizing the absorptance with a criterion of minimal standard deviation between layers (Fig. 5a, c: left columns). The primarily optimized target consisted of 288 nanorods in a $1 \times 1 \times 21 \mu \mathrm{m}^{3}$ supercell of polymer model-target and exhibited $\alpha_{\text {avg }}=948 \mathrm{~cm}^{-1}$ average absorption coefficient, $A_{\text {avg }}=0.863$ average absorptance with $\delta_{\text {avg }}=0.137$ average standard deviation.

First, a representative random location distribution exhibiting $\alpha=1175 \mathrm{~cm}^{-1}$ absorption coefficient, $A=0.915$ absorptance with $\delta=0.162$ that are slightly larger than the averaged values, was inspected in time-domain (Fig. 5a, c: right columns). The absorption coefficient and absorptance is in accordance with the expectation: the four-times larger number of nanorods with four-times smaller average $A C S$ results in almost the same values, as those achieved with the core-shell distribution.

There is an obvious difference between energy (power loss) and energy density (power loss density) in different neighboring layers, which increases over time, similarly to the core-shell distribution (Fig. 5ea, ec (ga, gc) and eb, ed (gb, gd)). At 240 fs delay corresponding to the time instant of complete overlap of the two counterpropagating pulses at the target center, the standard deviation of energy and power loss is $\delta_{\text {energy }}=\delta_{\text {power_loss }}=0.633$, whereas the standard deviation of energy and power loss density is $\delta_{\text {energy_density }}=\delta_{\text {power_los__density }}=0.535$, which indicate 3.9- and 3.3-fold increase in standard deviation compared to that of the absorptance distribution, respectively (Fig. 5ee (ge) and ef (gf)). There is no difference between tendencies of energy and power loss and of energy density and power loss density, since the complete energy is transformed into power loss in the compact metal nanorod (Fig. 5e-to-g). The energy and energy density distribution integrated in the complete layer exhibit a standard deviation of $\delta_{\text {energy_layer }}=\delta_{\text {energy_density_layer }}=0.218$ that is smaller compared to the multitude of nanoparticles, similarly to the core-shell layers (Fig. 5ee and ef). The decrease in standard 
deviation originates from the weaker dominance of the nanorod distribution.

The proposed nanorod distribution was determined by adjusting the previously optimized Gaussian number density distribution. The adjusted distribution exhibited an $\alpha_{\text {avg }}=731 \mathrm{~cm}^{-1}$ average absorption coefficient, $A_{\text {avg }}=0.785$ average absorptance with $\delta_{\text {avg }}=0.274$ average standard deviation of absorptance (Fig. 5b, d: left columns). A representative system achieved via the successive modification of the number density distribution along the layers made it possible to reach a higher $\alpha=987 \mathrm{~cm}^{-1}$ absorption coefficient and $A=0.874$ absorptance (Fig. 5 b, d: right columns). However, this can be achieved at the cost of almost four-times larger $\delta=0.605$ standard deviation of absorptance.

All inspected quantities exhibit global maxima similarly to those in the primarily optimized nanorod distribution, but these are less distinguishable in succeeding layers after nanorod distribution adjustment, similarly to the core-shell counterparts (Fig. 5fa, fb, ha, hb to ea, eb, ga, gb). The tendencies of integrated energy, power loss, and their corresponding densities in the successively approximated nanorod distribution are moderated with respect to those of primarily optimized ones, similarly to the counterpart core-shell distributions presented earlier (Fig. 5e-to-f and g-to-h). The only characteristic difference between the core-shell and nanorod distributions is that the power loss and power loss density exhibit the same tendencies as the energy and energy density in case of nanorods according to the proportionality of the energy and power loss in the solid nanoparticles; therefore, their standard deviation is analogous (Fig. 5e-to-g and f-to-h). Moreover, not only the tendencies, but the instantaneous and integrated values are also comparable for all of the distributions examined, despite the four-fold difference between the number densities of core-shell nanoparticles and nanorods. This again indicates the governing role of the nanoresonator ACS at a specific central wavelength. It seems to be counterintuitive that the corresponding energy and power loss density values are also close to each other, but this is the result of the five-times larger total core-shell volume. However, the difference in standard deviation stems only partially from the differences in the number density distributions along the layers; it is affected by the actual random nanoresonator positions inside the layers as well.

The considerable advantage of the successively adjusted nanorod number density distribution is that the standard deviation of energy and power loss (energy density and power loss density) is 7.7-times (2.5-times) smaller compared to the values in case of the primarily optimized distribution, namely, $\delta_{\text {energy }}=\delta_{\text {power_loss }}=0.082$ $\left(\delta_{\text {energy_density }}=\delta_{\text {power_loss_density }}=0.212\right)$ is achieved (Fig. 5fa, fc, fe (fb, fd, ff) and ha, hc, he (hb, hd, hf)).
The standard deviation of the energy (energy density) $\delta_{\text {energy_layer }}=\left(\delta_{\text {energy_density_layer }}\right)=0.198$ is larger (slightly smaller) for the complete layers compared to the multitude of nanoparticles caused by the energy capture inside and around the nanorods in case of the specific location distribution selected from the manifolds of adjusted intermediately narrow Gaussian number density distributions (Fig. 5fe and $\mathrm{ff})$. The standard deviation is smaller compared to the optimized distribution for the specific adjusted locations both for energy and energy density.

According to the central symmetry of the inspected core-shell nanoparticle and nanorod distributions, there is a negligible difference in either of the absorptance, energy (power loss), moreover in the energy density (power loss density) distributions in corresponding symmetrically aligned layers (Figs. 4 and 5).

A direct comparison of the energy, power loss, and their density distributions of the doped targets uncovers that while in case of the core-shell nanoparticles distributions of all these quantities show a minimum at the target center, only the adjusted nanorod distribution results in minima but only in the energy and power loss densities, which originate from normalization by the large total nanoresonator volume at the central layer (Fig. 4e, f, g, h/e, f and Fig. 5e, f, g, h/e, f). This shows that the exponential decay was gradually compensated in case of the inspected nanorod distributions. A maximum appears uniquely in the energy and energy density distributions of the complete layers in the optimized nanorod distribution, which is a reminiscence of the energy deposited in the multitude of nanoresonators. In the adjusted case, the usual exponential decrease is observed (Fig. 5fe and ff). For the adjusted nanorod distribution the absorption coefficient and the absorptance is just slightly smaller, whereas the standard deviation of absorptance is 6.6times larger; moreover, the energy standard deviation is 1.7times larger than the $\delta$ of the adjusted core-shell distribution. However, the standard deviation of energy density, power loss, and power loss density is 1.4-, 1.5-, and 1.8-times smaller in case of the optimized nanorod distribution.

\section{Charge Separation Study just Below the Damage Threshold}

Our results prove that the time-dependent charge separation computed via a hydrodynamic model is very similar to the charge separation determined by a Drude model, which confirms that the absorption and absorptance is almost linearly scaled with the intensity below the damage threshold of nanoresonators.

The $25 \mathrm{~nm} \times 75 \mathrm{~nm}$ resonant nanorod supports a charge oscillation, which reaches a maximum of $1.28 \mathrm{C} / \mathrm{m}^{2}(1.22$ $\mathrm{C} / \mathrm{m}^{2}$ ) in surface charge separation corresponding to a maximum of $2.30 \times 10^{8} \mathrm{C} / \mathrm{m}^{3}\left(2.21 \times 10^{8} \mathrm{C} / \mathrm{m}^{3}\right)$ in volume charge 
separation at $260.1 \mathrm{fs}$ ( $258.8 \mathrm{fs}$ ) based on the Drude (hydrodynamic model). The $25.5 \mathrm{~nm} / 30 \mathrm{~nm}$ resonant core-shell nanoparticle exhibits a charge oscillation of smaller amplitude, which reaches a maximum of $0.57 \mathrm{C} / \mathrm{m}^{2}\left(0.48 \mathrm{C} / \mathrm{m}^{2}\right)$ in outer surface charge separation corresponding to $1.47 \times 10^{8} \mathrm{C} / \mathrm{m}^{3}$ $\left(1.26 \times 10^{8} \mathrm{C} / \mathrm{m}^{3}\right)$ in volume charge separation at $256.1 \mathrm{fs}$ (256.0 fs) based on the Drude (hydrodynamic model).

\section{Conclusion}

Considering that the absorptance is orientation independent and significantly larger for the same number of nanoresonators, core-shell nanoparticles turned to be a better choice based on steady-state computations. Although the standard deviation of absorptance was larger for core-shell nanoparticles in the uniform and in different predefined Gaussian distributions, it became smaller in the primarily optimized distributions (except for energy density and power loss density). In case of the adjusted distributions, the standard deviation of the absorptance and energy was smaller for core-shell nanoresonators, but became larger for energy density as well as for power loss and power loss density in the nanoresonators, moreover for all quantities in the complete layers. The users' purposes define, whether core-shell nanoparticles possessing larger ACS and resulting in larger absorptance as well as smaller standard deviation of absorptance and deposited energy are preferred or nanorods, whose distributions exhibit a compromised absorptance and standard deviation of absorptance as well as deposited energy_- except in the adjusted distribution, when the complete layer is considered-but are more easily synthesizable via simple chemical procedures. The optimal configurations are experimentally realizable, since multilayers with micrometer scaled thicknesses embedding randomly distributed nanoresonators can be prepared via successive deposition techniques.

Acknowledgements The authors acknowledge the helpful discussions with Professor Pavel Ginzburg on hydrodynamic modeling of plasmonic nanostructures. B. Bánhelyi acknowledges support by the János Bolyai Research Scholarship of the Hungarian Academy of Sciences.

Emese Tóth acknowledges support by the project "Integrated program for training new generation of scientists in the fields of computer science" (EFOP-3.6.3-VEKOP-16-2017-00002). András Szenes acknowledges that this work was supported by the UNKP-20-3 New National Excellence Program of the Ministry for Innovation and Technology from the source of the National Research, Development and Innovation Fund.

Funding Open access funding provided by University of Szeged. This work was supported by the National Research, Development and Innovation Office (NKFIH) of Hungary, projects: "Optimized nanoplasmonics" (K116362), "Ultrafast physical processes in atoms, molecules, nanostructures and biological systems" (EFOP-3.6.2-16-2017-00005),
"Nanoplasmonic Laser Inertial Fusion Research Laboratory in the framework of the Hungarian National Laboratory program (NKFIH874-1/2020)," and the Eötvös Lóránd Research Network of Hungary.

Availability of Data and Material The proposed targets are numerically investigated by COMSOL simulation software.

\section{Declarations}

Ethics Approval This is a theoretical study, and no ethical approval is required.

Consent to Participate All authors agreed to participate in this research.

Consent for Publication Permission from all the authors has been taken to publish this manuscript.

Competing Interests The authors declare no competing interests.

Open Access This article is licensed under a Creative Commons Attribution 4.0 International License, which permits use, sharing, adaptation, distribution and reproduction in any medium or format, as long as you give appropriate credit to the original author(s) and the source, provide a link to the Creative Commons licence, and indicate if changes were made. The images or other third party material in this article are included in the article's Creative Commons licence, unless indicated otherwise in a credit line to the material. If material is not included in the article's Creative Commons licence and your intended use is not permitted by statutory regulation or exceeds the permitted use, you will need to obtain permission directly from the copyright holder. To view a copy of this licence, visit http://creativecommons.org/licenses/by/4.0/.

\section{References}

1. Prodan EP, Nordlander P (2004) Plasmon hybridization in spherical nanoparticles. J Chem Phys 120:5444-5454. https://doi.org/ $10.1063 / 1.1647518$

2. Halas NJ (2005) Playing with plasmons: tuning the optical resonant properties of metallic nanoshells. MRS Bull 30:362-367. https://doi.org/10.1557/mrs2005.99

3. Bardhan R, Grady NK, Ali T, Halas NJ (2010) Metallic nanoshells with semiconductor cores: optical characteristics modified by core medium properties. ACS Nano 4:6169-6179. https://doi.org/10. 1021/nn102035q

4. Tam F, Chen AL, Halas NJ (2007) Mesoscopic nanoshells: geometry-dependent plasmon resonances beyond the quasistatic limit. J Chem Phys 127:204703. https://doi.org/10.1063/1.2796169

5. Oldenburg SJ, Averitt RD, Westcott SL, Halas NJ (1998) Nanoengineering of optical resonances. Chem Phys Lett 288:243-247. https://doi.org/10.1016/S0009-2614(98)00277-2

6. Ni W, Kou X, Yang Z, Wang J (2008) Tailoring longitudinal surface plasmon wavelengths, scattering and absorption cross sections of gold nanorods. ACS Nano 2:677-686. https://doi.org/10. 1021/nn7003603

7. Juvé V, Cardinal MF, Lombardi A, Crut A, Maioli P, Pérez-Juste J, Liz-Marzán LM, Del Fatti N, Vellée F (2013) Size-dependent surface plasmon resonance broadening in nonspherical nanoparticles: single gold nanorods. Nano Lett 13:2234-2240. https://doi. org/10.1021/n1400777y 
8. Lal S, Link S, Halas NJ (2007) Nano-optics from sensing to waveguiding. Nat Photonics 1:641-648. https://doi.org/10.1038/ nphoton.2007.223

9. Zijlstra P, Paulo PMR, Orrit M (2012) Optical detection of single non-absorbing molecules using the surface plasmon resonance of a gold nanorod. Nat Nanotechnol 7:379-382. https://doi.org/10. 1038/nnano.2012.51

10. Szenes A, Bánhelyi B, Szabó LZs, Szabó G, Csendes T, Csete M, (2017) Improved emission of SiV diamond color centers embedded into concave plasmonic core-shell nanoresonators. Sci Rep 7:13845. https://doi.org/10.1038/s41598-017-14227-w

11. Szenes A, Bánhelyi B, Szabó LZs, Szabó G, Csendes T, Csete M, (2017) Enhancing diamond color center fluorescence via optimized plasmonic nanorod configuration. Plasmonics 12:12631280. https://doi.org/10.1007/s11468-016-0384-1

12. Gobin AM, Lee MH, Halas NJ, James WD, Drezek RA, West JL (2007) Near-infrared resonant nanoshells for combined optical imaging and photothermal cancer therapy. Nano Lett 7:1929_ 1934. https://doi.org/10.1021/n1070610y

13. Choi WI, Kim J-Y, Kang C, Byeon CC, Kim YH, Tae G (2011) Tumor regression in vivo by photothermal therapy based on goldnanorod-loaded, functional nanocarriers. ACS Nano 5:19952003. https://doi.org/10.1021/nn103047r

14. Baffou G, Cichos F, Quidant R (2020) Applications and challenges of thermoplasmonics. Nat Mater 19:946-958. https://doi.org/10. 1038/s41563-020-0740-6

15. Boriskina SV, Ghasemi H, Chen G (2013) Mater Today 16:375386. https://doi.org/10.1016/j.mattod.2013.09.003

16. Kern C, Zürch M, Petschulat J, Pertsch T, Kley B, Käsebier T, Hübner U, Spielmann C (2011) Comparison of femtosecond laser-induced damage on unstructured vs. nano-structured Au-targets. Appl Phys A 104:15-21. https://doi.org/10.1007/ s00339-011-6449-2

17. Nagy BJ, Zs P, Péter L, Prietl C, Krenn JR, Dombi P (2020) Near-field-induced femtosecond breakdown of plasmonic nanoparticles. Plasmonics 15:335-340. https://doi.org/10.1007/ s11468-019-01043-3

18. Plech A, Kotaidis V, Lorenc M, Boneberg J (2006) Femtosecond laser near-field ablation from gold nanoparticles. Nat Phys 2:44-47. https://doi.org/10.1038/nphys191

19. Rethfeld B, Kaiser A, Vicanek M, Simon G (2002) Ultrafast dynamics of nonequilibrium electrons in metals under femtosecond laser irradiation. Phy Rev B 65:214303. https://doi.org/10. 1103/PhysRevB.65.214303

20. Krasavin AV, Ginzburg P, Wurtz GA, Zayats AV (2016) Nonlocality-driven supercontinuum white light generation in plasmonic nanostructures. Nat Commun 7:11497. https://doi.org/10.1038/ ncomms 11497

21. Ginzburg P, Krasavin A, Sonnefraud Y, Murphy A, Pollard RJ, Maier SA, Zayats AV (2012) Nonlinearly coupled localized plasmon resonances: resonant second-harmonic generation. Phys Rev B 86:085422. https://doi.org/10.1103/PhysRevB.86.085422

22. Ginzburg P, Krasavin AV, Wurtz GA, Zayats AV (2015) Nonperturbative hydrodynamic model for multiple harmonics generation in metallic nanostructures. ACS Photonics 2:8-13. https://doi.org/ 10.1021/ph500362y

23. Scalora M, Vincenti MA, de Ceglia D, Roppo V, Centini M, Akozbek N, Bloemer MJ (2010) Second and third-harmonic generation in metal-based structures. Phys Rev A 82:043828. https:// doi.org/10.1103/PhysRevA.82.043828

24. Tanabe K (2016) Plasmonic energy nanofocusing for high-efficiency laser fusion ignition. Jpn J Appl Phys 55:08RG01. https://doi.org/10. 1109/MOC.2015.7416419

25. Tanabe K (2017) Plasmonic concepts for condensed matter nuclear fusion. J Condensed Matter Nucl Sci 24:296-300
26. Purvis MA, Shlyaptsev VN, Hollinger R, Bargsten C, Pukhov A, Prieto A, Wang Y, Luther BM, Yin L, Wang A, Rocca JJ (2013) Relativistic plasma nanophotonics for ultrahigh energy density physics. Nat Photonics 7:796-800. https://doi.org/10. 1038/nphoton.2013.217

27. Kaymak V, Pukhov A, Shlyaptsev VN, Rocca JJ (2016) Nanoscale ultradense Z-pinch formation from laser-irradiated nanowire arrays. Phys Rev Lett 117:035004. https://doi.org/10. 1103/PhysRevLett.117.035004

28. Bargsten C, Hollinger R, Capeluto MG, Kaymak V, Pukhov A, Wang S, Rockwood A, Wang Y, Keiss D, Tommasini R, London R, Park J, Busquet M, Klapisch M, Shlyaptsev VN, Rocca JJ (2017) Energy penetration into arrays of aligned nanowires irradiated with relativistic intensities: scaling to terabar pressures. Sci Adv 3:1601558. https://doi.org/10.1126/sciadv.1601558

29. Csernai LP, Csete M, Mishustin IN, Motornenko A, Papp I, Satarov LM, Stöcker H, Kroó N, Collaboration NAPLIFE (2020) Radiation-dominated implosion with flat target. Phys Wave Phenom 28:187-199. https://doi.org/10.3103/S1541308X20030048

30. Csernai LP, Kroó N, Papp I (2018) Radiation dominated implosion with nano-plasmonics. Laser Part Beams 36:171-178. https:// doi.org/10.1017/S0263034618000149

31. Papp I, Bravina L, Csete M, Mishustin IN, Molnár D, Motornenko A, Satarov LM, Stöcker H, Strottman DD, Szenes A, Vass D, Bíró TS, Csernai LP, Kroó N (2021) Laser wake field collider. Phys Lett A 396:127245. https://doi.org/10.1016/j.physleta.2021. 127245

32. Molesky S, Lin Z, Piggott AY, Jin W, Vuckovic J (2018) Inverse design in nanophotonics Nature Photon 12:659-670. https://doi. org/10.1038/s41566-018-0246-9

33. Milić M, Petković B, Selmi A, Dalibor P, Jermsittiparsert K, Radivojevic A, Milovancevic M, Khan A, Vidosavljevic ST, Denic N, Kuzman B (2021) Computational evaluation of microalgae biomass conversion to biodiesel. Biomass Conv Bioref. https://doi.org/10.1007/s13399-021-01314-2

34. Balogh J, Csendes T, Stateva RP (2003) Application of a stochastic method to the solution of the phase stability problem: cubic equations of state. Fluid Phase Equilibr 212:257-267. https://doi. org/10.1016/S0378-3812(03)00266-8

35. Csendes T, Pál L, Sendín JOH, Banga JR (2008) The GLOBAL optimization method revisited. Optim Lett 2:445-454. https://doi. org/10.1007/s11590-007-0072-3

36. Csete M, Szenes A, Vass D, Bánhelyi B, Dombi P (2020) Fewcycle localized plasmon oscillations Sci Rep 10:12986. https:// doi.org/10.1038/s41598-020-69761-x

37. Tóth B, Szenes A, Maráczi D, Bánhelyi B, Csendes T, Csete M (2020) Polarization independent high absorption efficiency single-photon detectors based on three-dimensional integrated superconducting and plasmonic patterns. IEEE J Sel Top Quant 26:3900309. https://doi.org/10.1109/JSTQE.2020.2987131

38. Petković D, Pavlović NT, Cojbašić Z (2016) Wind farm efficiency by adaptive neuro-fuzzy strategy. Int J Elec Power 81:215-221. https://doi.org/10.1016/j.ijepes.2016.02.020

39. Petković D (2017) Prediction of laser welding quality by computational intelligence approaches. Optik 140:597-600. https://doi. org/10.1016/j.ijleo.2017.04.088

40. Jović S, Danesh AS, Younesi E, Aničić O, Petković D, Shamshirband S (2016) Forecasting of underactuated robotic finger contact forces by support vector regression methodology. Int J Pattern Recogn 30:1659019. https://doi.org/10.1142/S0218001416590199

Publisher's Note Springer Nature remains neutral with regard to jurisdictional claims in published maps and institutional affiliations. 